\title{
Assessment of Inter-varietal Cross Lines of Gladiolus on Vegetative and Floral Traits for Selection and Promotion in Nepal
}

\author{
Tul Bahadur Poon ${ }^{1 *}$, Asmita Khanal ${ }^{1}$, SandipTimilsina ${ }^{1}$ and Anuja Rijal ${ }^{2}$ \\ ${ }^{1}$ Regional Agricultural Research Station, NARC, Lumle, Kaski \\ ${ }^{2}$ National Potato Research Program, NARC, Khumaltar, Lalitpur \\ "Corresponding Author's Email:chetpun2002@yahoo.co.in \\ "Orcid ID: https://orcid.org/0000-0003-2409-0365
}

Received on: 13 September 2020, Revised on: 16 April 2021, Accepted on: 21 April 2021

\begin{abstract}
Artificial crossing between species and varieties has been effective in producing new gladiolus cultivar with desirable traits. The seeds obtained from the total of twelve inter-varietal cross lines were raised in nurseries in 2013. The corms and cormels were selected yearly from the first phase to the fourth phase of selection. Forty seveninter-varietal cross lines and one check genotype were assessed in preliminary non-replicated trial in 2017 in the field of Regional Agricultural Research Station, Lumle, Nepal. A total of twenty five inter-varietal cross lines of gladiolus was selected, and assessed to develop new productive and attractive varieties of gladiolus on the basis of sixteen traits:the first spike emergence, complete spike emergence, the first floret loosening, maximum number of floret opening at a time, floret diameter, number of florets/spike, days to the seventh floret wilting, flower life, plant height, spike length, rachis length, number of spikes/mother corm, number of marketable spikes/mother corm, number of filled capsules/spike, number of unfilled capsules/spike and total number of capsules/spike. Forty six inter-varietal cross lines were selectivelyreduced to twenty five intervarietal cross lines after they had been assessed in the preliminary trial. Eventually ten cross lines: HRSDG013-2.17(A), HRSDG-013-7.4(B), HRSDG-013-7.8(A), HRSDG-013-7.8(B), HRSDG-013-8.5(A), HRSDG013-8.16(A), HRSDG-013-9.12(A-2), HRSDG-013-10.24, HRSDG-013-11.7(B) and HRSDG-013-11.17(B-2) are likely to be promoted for replicated trial so as to develop new desirable varieties in future.
\end{abstract}

Keywords: Artificial crossing, Desirable traits, Spikes, Rachis, Capsules.

\section{Introduction:}

Gladiolus is a genus of perennial herbaceous bulbous flowering plants of high economic importance. Cultivated gladiolus (Gladiolus hybridus Hort.) is a native of South Africa and Asia Minor belonging tothe family Iridaceae and Sub-family Ixiodeae. The genusGladiolusL. Ismostly tetraploid $(2 \mathrm{n}=4 \mathrm{x}=60)$, inter-specific hybrids being cultivated for more than 260 years (Goldblatt, 1996). The current number of species in the genus is 255 (Goldblatt and Manning, 1998). Among these, 180 species are originally from
South Africa (Duncan, 2000; Goldblatt, 1996; Goldblatt and Manning, 1998; Manning et al., 2002; Cantor andTolety, 2011).

Gladiolusis one of the most important among the bulbous ornamentals for cut flower trade in Nepal. The main emphasis in gladiolus improvement has been placed on development of varieties having attractive novel color and large number of florets mainly for cut flower, long spikes, more number of well spaced large sized florets, and good corm multiplication ability (Kumar and Yadav, 2005). 
Multiplication of planting material of gladiolus is most important because the cut flower trade of gladiolus is lagging behind over the recent years, owing to the unavailability of sufficient quality planting material at large scale (Barman et al., 2005). Moreover, new varieties also come from other countries, and the performances of these varieties depend upon climatic conditions of the region under which they are grown. As a result, cultivars, which perform well in one region, may not perform similarly in other regions of varying climatic conditions (Kamble et al., 2004). It is also important to develop desirable hybrids, and evaluate them with the existing cultivar for their superior desirable characters (Archana and Patil, 2013).

Nowadays, most of the gladiolus cultivars are developed from inter-specific and inter-varietal hybridization among several species and varieties. Hence, wide variation is exhibited among the cultivars for their growth, shape, spike length, floret traits and corm multiplication ability.

Artificial hybridization has also been very important in producing Gladiolus cultivars. The present-day very decorative garden varieties are the result of nearly 180 years of breeding and selection in Europe and North America, far away from South Africa, the native range of the original species (Ohriand and Khoshoo, 1983). Hybridization between wild species and cultivars of Gladiolus is an effective means of producing individuals(genotypes) withdesirablefeatures(flowerformand shape, color diversity or scent).

It is also important to develop Nepali hybrids and evaluate them with the existing cultivar for their superior desirable characters (Poon et al., 2012). Hence, the present preliminary experiment was conducted to discover the basic performances of newly evolved inter-varietal lines for different traits of gladiolus and also suitable for commercial cultivation in Nepal after being promoted and evaluated in replicated trials in station and on-farm conditions in the country.

\section{Materials and Methods:}

Present preliminary non-replicated trial was carried out with forty six inter-varietal cross lines and onecheck genotype of gladiolus that were obtained from the total of 12 inter-varietal crosses of gladiolus in 2013 in the field of Horticulture Research Station (HRS), Dailekh. Corms and cormels from the total of 200 and 125strains/ variants respectively in the first and second phase of selections were made and tagged for their identities depending upon type of crosses and variants within the crosses in 2014 and 2015 consecutively in the field of HRS, Dailekh. Similarly in the third phase of selection, only 75 variants were raised and only forty five variants were selected in the field of National Citrus Research Program, Dhankuta in 2016.

For the fourth phase of selection, twelvecorms and thirty cormelsfrom each of forty fiveinter-varietal cross lines (Variants) including onecheck genotype (ARSDG-04) were planted in two rows with corms and in one row with cormelsrespectively in 5 and 2.5 $\mathrm{cm}$ depth as per each experimental plot in February 12, 2017 in the field of Regional Agricultural Research station, Lumle, Kaski.The spacing of both corms and cormels between the rows were $50 \mathrm{~cm}$, and the distance of corms within the rows was $16.50 \mathrm{~cm}$, but the distance between cormels within the single row was only 3.3 $\mathrm{cm}$.Each of the $1.5 \mathrm{~m}^{2}(1.5 \mathrm{x} 1.0 \mathrm{~m})$ plot was incorporated with the basal application of $4.5 \mathrm{~kg}$ FYM (@30 t/ha), 33 g DAP (days after planting) (@39.60 kg Nitrogen/ha and 100 kg Phosphorus/ha),15 g Urea (@45.29 kg Nitrogen/ ha) and 37.50 g (@150 kg Potash/ha) prior to planting. Twelve corms and thirty cormelswere planted.

Remaining general intercultural operations were followed as per recommended for gladiolus production technology. The rest of 15 g Urea/plot (@45.29 kg Nitrogen/ha) was applied at the first side dressing in about 35 days of planting: The rest of 15 g Urea/plot (@ $45.29 \mathrm{~kg} \mathrm{Nitrogen} / \mathrm{ha}$ ) was applied at the second side dressing in about 60 days of planting.

On the basis of 16 traits : the first spike emergence, complete spike emergence, the first floret loosening, maximum number of floret opening at a time, floret diameter, number of florets /spike, days to the seventh floret wilting, flower life, plant height, spike length, rachis length, number of spikes/mother corm or hill, number of marketable spikes / mother corm or hill, number of filled capsules/spike, number of unfilled capsules/spike and total number of capsules/spike were assessed in preliminary non-replicated trial in accordance with the following ways.

Days to the first spike emergence: Days to the first spike emergence from the date of plantation of corms to the date of the first spike emergence was recorded from five labeled plants, and average was worked out. 
Days to complete spike emergence:Days to complete spike emergence from the date of plantation of corms to the date of full spike initiation was recorded from five labeled plants, and average was worked out.

Days to the first floret loosening: Number of days taken from plantation date of corms to the first floret unfurling/ opening /color breaking date was recorded from five labeled plants and average was worked out.

Maximum number of floret opening at a time (MNFO): It was recorded from five labeled plants when the first floret began to wither immediately after fully opening stage. Then, their average values were worked out.

Floret diameter $(\mathrm{cm})$ :Three basal florets diameter from each spike of five labeled plants was measured with vernier calipers, and average was worked out.

Number of florets /spike: Total number of florets per spike was counted from five labeled plants, and average value was worked out.

Days to the seventh floret wilting: Number of days taken from planting date of corms to the date of seventh floret wilting was counted from five labeled plants, and average vales were worked out.

Flower life (days) in field condition: Days taken from the date of the first floret loosening to the date of seventh floret wilting were recorded from five standing plants with labels, and average values were worked out so as to select promising inter-varietal cross lines for postharvest study in future.

Plant height $(\mathrm{cm})$ : The height of the five labeled plants was measured in each treatment plot from the soil surface to the tip of the plant with the last floret with the help of meter scale at the time of seventh floret wilting, and their average was worked out.

Spike length $(\mathrm{cm})$ : Its length was measured from base of spike to the top most floret at the time of complete opening of seventh floret. Then, the average spike length of five labeled plants was worked out.

Rachis length $(\mathrm{cm})$ :Length of rachis was measured from five spikes of labeled plants from the point of emergence of first floret to the last floret and expressed in centimeters.

Number of spikes/ mother corm or hill:The numbers of spikes were recorded from per hill (mother corm) from five labeled hill, and average was worked out.
Number of marketable spikes / mother corm or hill:The numbers of spikes were recorded from five labeled hill (mother corm), and average value was worked out.

Number of filled capsules/spike:It was recorded from the filled capsules /spike of five labeled plants, and average value was worked out.

Number of unfilled capsules/spike: It was recorded from the unfilled capsules /spike of five labeled plants, and average value was worked out.

Total number of capsules/spike: It was recorded from the total number of capsules /spike of five labeled plants, and average value was worked out.

\section{Results:}

Two vegetative traits viz., days to the first spike emergence and days to the complete spike emergence ; and two floret traits viz., days to the first floret loosening (DFFL), maximum number of floret opening (MNFO) at a time are presented in Table 1.

Days to the first spike emergence: It was variable between 91.75 days and 125.00 days with the mean value of 107.36 days. Nine inter-varietal cross lines and one check genotype were able to reveal the first spike emergence in fewer than 100 days after planting while three intervarietal cross lines were found to be late taking more than 120 days to show the first spike emergence. On the other hand, thirty four inter-varietal cross lines were mediocre to show the first spike emergence between 100 and 120 days after planting. When compared to all forty six cross lines, check genotype (ARSDG-04) with 97.12 days did not show such an earliness that four cross lines viz.,HRSDG2013-8.23(A) with 91.75 days, HRSDG-2013-11.21 with 95.75 days), HRSDG-2013-6.5(A-2) with 95.40 days and HRSDG-2013-6.7(B) with 97.00 days were somewhat earlier than that of check genotype (ARSDG-04). The remaining forty two cross lines were such late ones that check genotype (ARSDG-04) was far early in this trait as shown in Table 1.

Days to the complete spike emergence: it varied from 99 days to 129 days with the mean value of 110.00 days. Nine inter-varietal cross lines and one check genotype (ARSDG-04) were able to reveal the full spike emergence in fewer than 105 days after planting while one inter-varietal cross line viz., HRSDG -2013-9.16 (A) was found to be late taking more than 125 days to show 
complete spike emergence. On the other hand, thirty five inter-varietal cross lines were mediocre to show the complete spike emergence between 105 and 125 days after planting. As comparing to all forty six cross lines, Check genotype (ARSDG-04) with 101.00 days was earlier than three inter-varietal cross lines viz., HRSDG2013-8.23(A) with 99.00 days, HRSDG-2013-11.21 with 100.00 days) and HRSDG -2013-6.5(A-2) with 100.60 days but HRSDG-2013-1.2(A) with 101.00 days was similar to Check genotype (ARSDG-04) with 101.00 days, and it was a little later than HRSDG-2013-6.7(B) with 101.40 days. The remaining forty two cross lines were such late ones that check genotype (ARSDG-04) was far early in this trait in Table 1.

Days to the first floret loosening: It differed from 106.97 days to 134.00 days having the mean value of 114.45 days. Six inter-varietal cross lines showed the first floret loosening (DFFL) in fewer than 110 days after planting while five inter-varietal cross lines showed the first floret loosening (DFFL) in more than 125 days after planting. The rest thirty five inter-varietal cross lines and one check genotype (ARSDG-04) were mediocre for the same trait revealing between 110 days and
125 days. Five cross lines viz., HRSDG-2013-11.21 with 106.50 days, HRSDG-2013-6.5 (A-2) with 107.20 days, HRSDG-2013-6.7(B) with 107.40 days, HRSDG-2013-1.2(A) with 107.80 days and HRSDG2013-8.5(A) with 108.00 days recorded the little earlier than ARSDG-04 (110.25 days).The rest forty cross lines were so late that ARSDG-04 was quite earlier than them (Table 1).

Maximum number of floret opening at a time (MNFO): it was found difference between 5.50 and 10.10 with the mean value of 7.50.A total of fourteen inter-varietal cross lines produced more than 8.00 floret opening at a time (MNFO) whereas only two inter-varietal cross lines produced fewer than 6.00 floret opening at a time(MNFO). The rest of thirty inter-varietal cross lines produced between 6.00 and 8.00 floret opening at a time (MNFO).The fewest number of floret opening (5.50) at a time was found in HRSDG-2013-1.2 (B) and check genotype (ARSDG-04) whereas the remaining forty five cross lines recorded the greater number of floret opening at a time than that of check genotype (ARSDG-04) as presented in Table 1.

Table 1: Performance of 46 inter varietal crossed lines and check genotype of gladiolus for days to the first spike emergence, days to the full spike emergence, days to the first floret loosening (DFFL) and maximum number of floret opening at a time (MNFO) in 2017/2018 at RARS, Lumle.

\begin{tabular}{|c|c|c|c|c|c|}
\hline $\begin{array}{c}\text { Plot } \\
\text { number }\end{array}$ & Inter-varietal cross lines & $\begin{array}{c}\text { Days to the } \\
\text { first spike } \\
\text { emergence }\end{array}$ & $\begin{array}{c}\text { Days to the } \\
\text { complete spike } \\
\text { emergence }\end{array}$ & DFFL & MNFO \\
\hline 1 & HRSDG-2013-1.2(A) & 99.20 & 101.00 & 107.80 & 6.10 \\
\hline 2 & HRSDG-2013-1.2(B) & 104.40 & 108.00 & 114.20 & 5.50 \\
\hline 3 & HRSDG-2013-1.6(D) & 105.80 & 109.00 & 116.60 & 8.30 \\
\hline 4 & HRSDG-2013-1.14(B) & 100.00 & 104.00 & 110.00 & 7.00 \\
\hline 5 & HRSDG-2013-1.14(D) & 103.40 & 107.00 & 114.80 & 7.54 \\
\hline 6 & HRSDG-2013-1.8(D) & 105.00 & 109.00 & 114.00 & 8.25 \\
\hline 7 & HRSDG-2013-2.15(C) & 103.00 & 107.00 & 113.80 & 6.60 \\
\hline 8 & HRSDG-2013-2.13(A) & 102.40 & 106.60 & 114.40 & 8.00 \\
\hline 9 & HRSDG-2013-2.4(D) & 98.20 & 103.00 & 110.00 & 7.00 \\
\hline 10 & HRSDG-2013-2.17(A) & 106.00 & 109.00 & 117.70 & 8.25 \\
\hline 11 & HRSDG-2013-6.17(E) & 109.00 & 113.40 & 118.20 & 7.00 \\
\hline 12 & HRSDG-2013-6.5(A-2) & 95.40 & 100.60 & 107.20 & 8.60 \\
\hline 13 & HRSDG-2013-6.7(A) & 107.00 & 111.00 & 118.20 & 7.90 \\
\hline 14 & HRSDG-2013-6.7(B) & 97.00 & 101.40 & 107.40 & 6.10 \\
\hline 15 & HRSDG-2013-6.5(A-1) & 106.00 & 109.60 & 116.00 & 7.20 \\
\hline 16 & HRSDG-2013-7.4(B) & 106.00 & 109.00 & 118.20 & 7.90 \\
\hline
\end{tabular}




\begin{tabular}{|c|c|c|c|c|c|}
\hline $\begin{array}{c}\text { Plot } \\
\text { number }\end{array}$ & Inter-varietal cross lines & $\begin{array}{l}\text { Days to the } \\
\text { first spike } \\
\text { emergence } \\
\end{array}$ & $\begin{array}{c}\text { Days to the } \\
\text { complete spike } \\
\text { emergence }\end{array}$ & DFFL & MNFO \\
\hline 17 & HRSDG-2013-7.25(A) & 103.00 & 106.40 & 115.40 & 6.10 \\
\hline 18 & HRSDG-2013-7.8(A) & 98.00 & 103.00 & 112.00 & 7.20 \\
\hline 19 & HRSDG-2013-7.8(B) & 102.00 & 108.0 & 117.00 & 7.20 \\
\hline 20 & HRSDG-2013-8.23(A) & 91.75 & 99.00 & 119.80 & 6.40 \\
\hline 21 & HRSDG-2013-8.16(B) & 100.00 & 109.50 & 121.50 & 6.50 \\
\hline 22 & HRSDG-2013-8.5(B) & 98.40 & 102.40 & 109.00 & 8.00 \\
\hline 23 & HRSDG-2013-8.5(A) & 97.60 & 102.60 & 108.00 & 8.50 \\
\hline 24 & HRSDG-2013-8.16(A) & 115.00 & 119.00 & 125.40 & 8.60 \\
\hline 25 & HRSDG-2013-8.22(A) & 112.60 & 115.60 & 122.00 & 7.25 \\
\hline 26 & HRSDG-2013-9.12(A-2) & 101.20 & 105.40 & 113.20 & 7.00 \\
\hline 27 & HRSDG-2013-9.12(A) & 102.00 & 105.40 & 113.60 & 6.50 \\
\hline 28 & HRSDG-2013-9.3(D) & 104.00 & 108.00 & 114.00 & 7.30 \\
\hline 29 & HRSDG-2013-9.22(A) & 106.00 & 109.00 & 114.20 & 8.80 \\
\hline 30 & HRSDG-2013-9.7(A) & 114.00 & 111.00 & 123.60 & 8.80 \\
\hline 31 & HRSDG-2013-9.22(E) & 121.00 & 124.00 & 129.20 & 7.50 \\
\hline 32 & HRSDG-2013-9.16(A) & 125.00 & 129.00 & 134.00 & 6.50 \\
\hline 33 & HRSDG-2013-10.24 & 116.00 & 119.00 & 124.70 & 7.10 \\
\hline 34 & HRSDG-2013-10.4 & 111.00 & 114.40 & 121.40 & 5.80 \\
\hline 35 & HRSDG-2013-10.18 & 108.50 & 112.25 & 117.70 & 8.10 \\
\hline 36 & HRSDG-2013-10.5(A) & 117.50 & 120.75 & 127.60 & 6.60 \\
\hline 37 & HRSDG-2013-10.45 & 109.00 & 113.20 & 121.80 & 6.40 \\
\hline 38 & HRSDG-2013-11.2(B) & 104.30 & 108.00 & 114.20 & 8.00 \\
\hline 39 & HRSDG-2013-11.37(B) & 107.60 & 111.20 & 118.00 & 10.10 \\
\hline 40 & HRSDG-2013-11.33 & 101.00 & 105.00 & 111.40 & 7.70 \\
\hline 41 & HRSDG-2013-11.17(B-1) & 105.60 & 109.40 & 115.00 & 8.10 \\
\hline 42 & HRSDG-2013-11.17(B-2) & 104.00 & 108.00 & 113.80 & 7.90 \\
\hline 43 & HRSDG-2013-11.7(B) & 106.00 & 109.00 & 116.80 & 8.70 \\
\hline 44 & HRSDG-2013-11.21 & 95.25 & 100.00 & 106.50 & 6.00 \\
\hline 45 & HRSDG-2013-11.4(B) & 103.25 & 107.00 & 114.50 & 8.20 \\
\hline 46 & HRSDG-2013-11.42 & 126.50 & 130.50 & 136.50 & 9.50 \\
\hline 47 & ARSDG-04/Check & 97.12 & 101.00 & 110.25 & 5.50 \\
\hline \multicolumn{2}{|r|}{ Minimum } & 91.75 & 99.00 & 106.97 & 5.50 \\
\hline \multicolumn{2}{|r|}{ Mean } & 107.36 & 110.00 & 114.45 & 7.50 \\
\hline \multicolumn{2}{|r|}{ Maximum } & 125.00 & 129.00 & 134.00 & 10.10 \\
\hline \multicolumn{2}{|r|}{$\mathrm{CV} \%$} & 14.71 & 6.071 & 14.80 & 13.70 \\
\hline
\end{tabular}

Four floret traits viz. spike length, rachis length, floret diameter and number of florets /spike is presented in Table 2.

Spike length (cm): It differed from $82.30 \mathrm{~cm}$ to 131.00 $\mathrm{cm}$ with the mean value of $106.70 \mathrm{~cm}$. A total of 18 intervarietal cross lines exhibited the spike length above 110 cm while 12 inter-varietal cross lines and one check genotype (ARSDG-04) having the spike length below
$100.00 \mathrm{~cm}$. The rest of 16 inter-varietal cross lines had the spike length between 100.00 and $110.00 \mathrm{~cm}$. Twelve cross lines : HRSDG-2013- 9.7 (A) with $(82.30 \mathrm{~cm})$, HRSDG-2013- 9.22 (E) with (84.80cm),HRSDG-20139.22 with $(87.60 \mathrm{~cm}), \mathrm{HRSDG}-2013-8.16$ (B) with $(88.50 \mathrm{~cm})$, HRSDG-2013- 1.6 (D) with $(88.50 \mathrm{~cm})$, HRSDG-2013- 8.16 (B) with (88.50cm),HRSDG-20136.2 (A-2) with $(88.80 \mathrm{~cm})$, HRSDG-2013- 8.23 (A) with 
(91.48cm), HRSDG-2013- 1.2 (A) with $(93.60 \mathrm{~cm})$ HRSDG-2013- 8.5 (A) with (94.00 cm) HRSDG-201311.2(B) with (94.25 cm) and HRSDG-2013- 9.12 (A) with $(94.60 \mathrm{~cm})$ reflected shorter spike length than that of check genotype (ARSDG-04) with $96.00 \mathrm{~cm}$. The rest of thirty four cross lines produced longer spikes than that of check genotype (ARSDG-04).
Rachis length (cm): It differed from $54.30 \mathrm{~cm}$ to 108.00 $\mathrm{cm}$ with the mean value of $72.52 \mathrm{~cm}$. A total of 17 inter-varietal cross lines exhibited the rachis length above $75.00 \mathrm{~cm}$ while 12 inter-varietal cross lines having the spike length below $65.00 \mathrm{~cm}$. The rest of 17 inter-varietal cross lines and one check genotype (ARSDG-04) had the spike length between 65.00 and $75.00 \mathrm{~cm}$.

Table 2: Performance of 46 inter varietal crossed lines and check genotype of gladiolus for days to spike length, rachis length, floret diameter and number of florets/spike in 2017/2018 at RARS, Lumle.

\begin{tabular}{|c|c|c|c|c|c|}
\hline $\begin{array}{c}\text { Plot } \\
\text { number }\end{array}$ & Inter-varietal cross lines & $\begin{array}{c}\text { spike } \\
\text { length }(\mathrm{cm})\end{array}$ & $\begin{array}{c}\text { Rachis } \\
\text { length }(\mathrm{cm})\end{array}$ & $\begin{array}{c}\text { Floret } \\
\text { diameter }(\mathrm{cm})\end{array}$ & $\begin{array}{l}\text { Number of } \\
\text { florets/spike }\end{array}$ \\
\hline 1 & HRSDG-2013-1.2(A) & 93.60 & 66.40 & 7.70 & 13.80 \\
\hline 2 & HRSDG-2013-1.2(B) & 98.40 & 70.60 & 7.76 & 13.25 \\
\hline 3 & HRSDG-2013-1.6(D) & 88.80 & 61.20 & 6.92 & 13.50 \\
\hline 4 & HRSDG-2013-1.14(B) & 102.66 & 66.00 & 8.75 & 14.60 \\
\hline 5 & HRSDG-2013-1.14(D) & 92.00 & 66.80 & 7.50 & 15.40 \\
\hline 6 & HRSDG-2013-1.8(D) & 123.50 & 84.50 & 8.85 & 14.00 \\
\hline 7 & HRSDG-2013-2.15(C) & 109.60 & 77.40 & 8.08 & 16.00 \\
\hline 8 & HRSDG-2013-2.13(A) & 108.25 & 73.25 & 8.71 & 15.30 \\
\hline 9 & HRSDG-2013- 2.4(D) & 112.80 & 68.80 & 8.07 & 15.60 \\
\hline 10 & HRSDG-2013-2.17(A) & 108.20 & 78.20 & 8.78 & 18.75 \\
\hline 11 & HRSDG-2013-6.17(E) & 118.50 & 79.20 & 9.12 & 18.00 \\
\hline 12 & HRSDG-2013-6.5 (A-2) & 98.80 & 56.60 & 10.60 & 15.25 \\
\hline 13 & HRSDG-2013-6.7(A) & 102.60 & 70.60 & 9.16 & 15.50 \\
\hline 14 & HRSDG-2013-6.7(B) & 123.20 & 77.40 & 9.30 & 12.50 \\
\hline 15 & HRSDG-2013-6.5(A-1) & 105.80 & 63.80 & 9.72 & 16.00 \\
\hline 16 & HRSDG-2013-7.4(B) & 102.60 & 70.60 & 9.50 & 12.80 \\
\hline 17 & HRSDG-2013-7.25(A) & 123.20 & 77.40 & $8 . .15$ & 14.30 \\
\hline 18 & HRSDG-2013-7.8(A) & 105.80 & 63.80 & 8.93 & 14.28 \\
\hline 19 & HRSDG-2013-7.8(B) & 106.00 & 89.00 & 6.42 & 19.50 \\
\hline 20 & HRSDG-2013-8.23(A) & 91.48 & 63.88 & 8.80 & 14.25 \\
\hline 21 & HRSDG-2013-8.16(B) & 88.50 & 64.50 & 8.27 & 14.50 \\
\hline 22 & HRSDG-2013-8.5(B) & 105.60 & 76.40 & 8.54 & 16.40 \\
\hline 23 & HRSDG-2013-8.5(A) & 94.00 & 59.60 & 8.55 & 15.23 \\
\hline 24 & HRSDG-2013-8.16(A) & 100.80 & 69.20 & 8.54 & 17.20 \\
\hline 25 & HRSDG-2013-8.22(A) & 112.50 & 75.00 & 8.47 & 14.25 \\
\hline 26 & HRSDG-2013-9.12(A-2) & 118.00 & 77.60 & 7.56 & 15.00 \\
\hline 27 & HRSDG-2013-9.12(A) & 94.60 & 67.20 & 7.23 & 16.60 \\
\hline 28 & HRSDG-2013-9.3(D) & 111.67 & 76.30 & 7.03 & 14.33 \\
\hline 29 & HRSDG-2013-9.22(A) & 87.60 & 57.60 & 8.69 & 15.80 \\
\hline 30 & HRSDG-2013-9.7(A) & 82.30 & 54.30 & 6.63 & 13.60 \\
\hline 31 & HRSDG-2013-9.22(E) & 84.80 & 63.00 & 7.60 & 15.75 \\
\hline
\end{tabular}




\begin{tabular}{|c|c|c|c|c|c|}
\hline $\begin{array}{c}\text { Plot } \\
\text { number }\end{array}$ & Inter-varietal cross lines & $\begin{array}{c}\text { spike } \\
\text { length } \mathbf{( c m})\end{array}$ & $\begin{array}{c}\text { Rachis } \\
\text { length } \mathbf{( c m})\end{array}$ & $\begin{array}{c}\text { Floret } \\
\text { diameter }(\mathbf{c m})\end{array}$ & $\begin{array}{c}\text { Number of } \\
\text { florets/spike }\end{array}$ \\
\hline 32 & HRSDG-2013-9.16(A) & 116.60 & 71.30 & 7.28 & 14.60 \\
\hline 33 & HRSDG-2013-10.24 & 114.50 & 57.75 & 8.81 & 15.75 \\
\hline 34 & HRSDG-2013-10.4 & 100.80 & 78.00 & 5.86 & 14.75 \\
\hline 35 & HRSDG-2013-10.18 & 121.00 & 90.60 & 7.98 & 18.00 \\
\hline 36 & HRSDG-2013-10.5(A) & 106.50 & 61.50 & 7.30 & 14.50 \\
\hline 37 & HRSDG-2013-10.45 & 114.00 & 90.00 & 7.72 & 15.80 \\
\hline 38 & HRSDG-2013-11.2(B) & 94.25 & 65.50 & 8.36 & 12.75 \\
\hline 39 & HRSDG-2013-11.37(B) & 111.40 & 71.60 & 9.34 & 18.40 \\
\hline 40 & HRSDG-2013-11.33 & 109.40 & 77.20 & 8.30 & 14.20 \\
\hline 41 & HRSDG-2013-11.17(B-1) & 110.70 & 75.50 & 10.80 & 15.00 \\
\hline 42 & HRSDG-2013-11.17(B-2) & 108.40 & 70.00 & 10.20 & 15.60 \\
\hline 43 & HRSDG-2013-11.7(B) & 142.20 & 108.00 & 8.80 & 18.20 \\
\hline 44 & HRSDG-2013-11.21 & 120.00 & 73.30 & 7.45 & 13.50 \\
\hline 45 & HRSDG-2013-11.4(B) & 120.50 & 71.50 & 6.60 & 13.50 \\
\hline 46 & HRSDG-2013-11.42 & 131.00 & 82.50 & 9.25 & 17.50 \\
\hline 47 & ARSDG-04/Check & 96.00 & 71.00 & 7.20 & 13.00 \\
\hline & Minimum & 82.30 & 54.30 & 5.56 & 12.50 \\
\hline & Mean & 106.70 & 72.52 & 8.30 & 15.30 \\
\hline & Maximum & 131.00 & 108.00 & 10.80 & 19.50 \\
\hline & CV\% & 11.92 & 14.69 & 12.81 & 10.91 \\
\hline
\end{tabular}

Floret Diameter $(\mathrm{cm})$ : it ranged from $5.56 \mathrm{~cm}$ to 10.80 $\mathrm{cm}$ with the mean value of $8.30 \mathrm{~cm}$. A total of 9 intervarietal cross lines exhibited the floret diameter above $9.00 \mathrm{~cm}$ while 17 inter-varietal cross lines and one check genotype (ARSDG-04) had the floret diameter below $8.00 \mathrm{~cm}$. The rest of 20 inter-varietal cross lines had the floret diameter between 8.00 and $9.00 \mathrm{~cm}$. Only six cross lines: ARSDG-2013-10.4 (5.86 cm), ARSDG-2013-7.8 (B) with $6.42 \mathrm{~cm}$, ARSDG-2013-11.4 (B) with $6.60 \mathrm{~cm}$, ARSDG-2013-9.7 (A) with $6.63 \mathrm{~cm}$, ARSDG-2013-1.6 (D) with $6.92 \mathrm{~cm}$ and ARSDG-2013-9.3 (D) with 7.03 $\mathrm{cm}$ produced narrower floret diameters than that of check genotype (ARSDG-04) with $7.20 \mathrm{~cm}$.

Number of florets/spike:The fewest number of florets (12.50/spike) and the highest number of florets (19.50/ spike) were recorded with the mean value of 15.30 / spike due to the effect of 46 inter-varietal cross lines and one check genotype (ARSDG-04). The maximum number of florets with more than 17/spike were recorded in 8 inter-varietal cross lines whereas the minimum number of florets fewer than 14 /spike were recorded in 9 inter-varietal cross lines. A total of 29 inter-varietal cross lines recorded the number of florets /spike that ranged between 14 and 17. Only three cross lines viz., HRSDG-2013-6.7 (B) with $12.50 \mathrm{~cm}$, HRSDG-2013-11.2 (B) with $12.75 \mathrm{~cm}$ and HRSDG-2013-7.4 (B) with $12.80 \mathrm{~cm}$ produced smaller number of florets/spike than that of check genotype (ARSDG-04) with 13.00 florets /spike.One vegetative trait (plant height) and three floral traits viz., number of spikes/ mother corm, number of marketable spikes/ mother corm and days to the seventh floret wilting are presented in Table 3.

Plant height: It differed from $94.00 \mathrm{~cm}$ to $160.00 \mathrm{~cm}$ with the mean value of $116.00 \mathrm{~cm}$. A total of 11 intervarietal cross lines produced the plant heights with more than 130.00 while nine inter-varietal cross lines produced plant heights with lower than $110.00 \mathrm{~cm}$. The remaining 26 inter-varietal cross lines and one check genotype (ARSDG-04) produced plant heights measuring between $110.00 \mathrm{~cm}$ and $130.00 \mathrm{~cm}$. A total of twenty eight cross lines produced shorter plant height than that of check genotype (ARSDG-04) with $125 \mathrm{~cm}$ while the rest of eighteen cross lines produced taller plants than that of check genotype (ARSDG-04). 
Number of spikes /mother corm or hill: It was found different between 1.00 and 5.00 with the mean value of 2.76. Twelve inter-varietal cross lines produced more than 3 spikes/mother corm whilst ten inter-varietal cross lined produced fewer than 2 spikes/mother corm. The rest 24 inter-varietal cross lines and one check genotype (ARSDG-04) produced the spike numbers between 2 and 3 /mother corm .A total thirty one cross lines produced high number of spikes/mother corm as compared to that of check genotype (ARSDG-04) with 2.00 spikes/corm.

Table 3: Performance of 46 inter varietal crossed lines and check genotype of gladiolus for plant height, number of spikes/mother corm, number of marketable spikes/ mother corm and days to the seventh floret wilting in 2017/2018 at RARS, Lumle.

\begin{tabular}{|c|c|c|c|c|c|}
\hline $\begin{array}{c}\text { Plot } \\
\text { number }\end{array}$ & Inter-varietal cross lines & $\begin{array}{l}\text { Plant height } \\
\text { (cm) }\end{array}$ & $\begin{array}{l}\text { Number of } \\
\text { spikes/mother } \\
\text { corm }\end{array}$ & $\begin{array}{l}\text { Number of mar- } \\
\text { ketable spikes/ } \\
\text { mother corm }\end{array}$ & $\begin{array}{c}\text { Days to the } \\
\text { seventh } \\
\text { floret } \\
\text { wilting }\end{array}$ \\
\hline 1 & HRSDG-2013-1.2(A) & 110.00 & 5.00 & 3.50 & 116.80 \\
\hline 2 & HRSDG-2013-1.2(B) & 117.00 & 2.75 & 2.75 & 123.20 \\
\hline 3 & HRSDG-2013-1.6(D) & 105.00 & 3.75 & 2.50 & 126.00 \\
\hline 4 & HRSDG-2013-1.14(B) & 122.00 & 2.00 & 1.60 & 120.30 \\
\hline 5 & HRSDG-2013-1.14(D) & 106.80 & 3.20 & 3.00 & 123.00 \\
\hline 6 & HRSDG-2013-1.8(D) & 107.00 & 2.00 & 1.25 & 121.75 \\
\hline 7 & HRSDG-2013-2.15(C) & 125.20 & 2.80 & 2.20 & 121.80 \\
\hline 8 & HRSDG-2013-2.13(A) & 132.00 & 1.75 & 1.75 & 123.40 \\
\hline 9 & HRSDG-2013- 2.4(D) & 123.00 & 3.00 & 2.75 & 117.20 \\
\hline 10 & HRSDG-2013-2.17(A) & 124.20 & 2.60 & 2.20 & 125.70 \\
\hline 11 & HRSDG-2013-6.17(E) & 123.14 & 2.50 & 2.00 & 126.20 \\
\hline 12 & HRSDG-2013-6.5 (A-2) & 115.20 & 3.50 & 2.50 & 115.20 \\
\hline 13 & HRSDG-2013-6.7(A) & 125.25 & 1.60 & 1.60 & 125.80 \\
\hline 14 & HRSDG-2013-6.7(B) & 145.70 & 5.00 & 3.60 & 115.20 \\
\hline 15 & HRSDG-2013-6.5(A-1) & 120.00 & 3.00 & 2.60 & 124.50 \\
\hline 16 & HRSDG-2013-7.4(B) & 118.20 & 3.00 & 1.60 & 128.20 \\
\hline 17 & HRSDG-2013-7.25(A) & 142.60 & 3.25 & 2.50 & 122.65 \\
\hline 18 & HRSDG-2013-7.8(A) & 136.40 & 2.60 & 2.30 & 120.57 \\
\hline 19 & HRSDG-2013-7.8(B) & 142.50 & 2.50 & 2.25 & 126.00 \\
\hline 20 & HRSDG-2013-8.23(A) & 105.20 & 4.20 & 3.00 & 128.00 \\
\hline 21 & HRSDG-2013-8.16(B) & 96.50 & 2.00 & 1.60 & 129.50 \\
\hline 22 & HRSDG-2013-8.5(B) & 120.20 & 2.40 & 1.80 & 117.20 \\
\hline 23 & HRSDG-2013-8.5(A) & 108.00 & 4.50 & 3.20 & 116.60 \\
\hline 24 & HRSDG-2013-8.16(A) & 112.80 & 1.00 & 1.00 & 135.00 \\
\hline 25 & HRSDG-2013-8.22(A) & 119.25 & 3.00 & 2.50 & 130.75 \\
\hline 26 & HRSDG-2013-9.12(A-2) & 136.20 & 4.00 & 2.30 & 122.70 \\
\hline 27 & HRSDG-2013-9.12(A) & 107.80 & 2.40 & 1.60 & 120.60 \\
\hline 28 & HRSDG-2013-9.3(D) & 121.66 & 4.00 & 3.00 & 123.60 \\
\hline 29 & HRSDG-2013-9.22(A) & 100.20 & 3.80 & 2.40 & 123.45 \\
\hline 30 & HRSDG-2013-9.7(A) & 94.00 & 2.00 & 1.60 & 132.30 \\
\hline
\end{tabular}




\begin{tabular}{|c|c|c|c|c|c|}
\hline $\begin{array}{c}\text { Plot } \\
\text { number }\end{array}$ & Inter-varietal cross lines & $\begin{array}{l}\text { Plant height } \\
\text { (cm) }\end{array}$ & $\begin{array}{l}\text { Number of } \\
\text { spikes/mother } \\
\text { corm }\end{array}$ & $\begin{array}{l}\text { Number of mar- } \\
\text { ketable spikes/ } \\
\text { mother corm }\end{array}$ & $\begin{array}{l}\text { Days to the } \\
\text { seventh } \\
\text { floret } \\
\text { wilting }\end{array}$ \\
\hline 31 & HRSDG-2013-9.22(E) & 118.70 & 1.50 & 1.20 & 139.20 \\
\hline 32 & HRSDG-2013-9.16(A) & 127.00 & 3.00 & 2.00 & 141.30 \\
\hline 33 & HRSDG-2013-10.24 & 126.00 & 2.20 & 2.20 & 133.20 \\
\hline 34 & HRSDG-2013-10.4 & 124.50 & 2.20 & 1.80 & 131.00 \\
\hline 35 & HRSDG-2013-10.18 & 139.00 & 2.75 & 2.60 & 126.70 \\
\hline 36 & HRSDG-2013-10.5(A) & 117.50 & 1.50 & 1.50 & 137.10 \\
\hline 37 & HRSDG-2013-10.45 & 160.00 & 1.60 & 1.60 & 132.40 \\
\hline 38 & HRSDG-2013-11.2(B) & 113.25 & 1.60 & 1.60 & 122.50 \\
\hline 39 & HRSDG-2013-11.37(B) & 123.60 & 1.30 & 1.30 & 126.00 \\
\hline 40 & HRSDG-2013-11.33 & 122.40 & 1.70 & 1.70 & 119.20 \\
\hline 41 & HRSDG-2013-11.17(B-1) & 123.00 & 2.50 & 2.50 & 123.50 \\
\hline 42 & HRSDG-2013-11.17(B-2) & 127.80 & 4.50 & 2.25 & 121.13 \\
\hline 43 & HRSDG-2013-11.7(B) & 157.20 & 1.40 & 1.40 & 124.00 \\
\hline 44 & HRSDG-2013-11.21 & 125.50 & 2.00 & 1.75 & 115.50 \\
\hline 45 & HRSDG-2013-11.4(B) & 134.70 & 2.20 & 2.20 & 122.25 \\
\hline 46 & HRSDG-2013-11.42 & 140.50 & 2.00 & 2.00 & 145.00 \\
\hline 47 & ARSDG-04/Check & 125.00 & 2.00 & 1.50 & 119.00 \\
\hline & Minimum & 94.00 & 1.00 & 1.00 & 115.00 \\
\hline & Mean & 116.00 & 2.76 & 2.12 & 125.15 \\
\hline & Maximum & 160.00 & 5.00 & 3.60 & 141.00 \\
\hline & $\mathrm{CV} \%$ & 17.86 & 44.36 & 28.88 & 5.49 \\
\hline
\end{tabular}

Number of marketable spikes/ mother corm or hill: It differed from 1.00 to 3.6 with mean value of 2.12 spike /mother corm. Nine inter-varietal cross lines showed more than 2.50 marketable spikes/mother corm, but only five inter-varietal cross lines showed fewer than 1.5 marketable spikes/mother corms. Thirty two, the highest number of inter-varietal cross lines and one check genotype (ARSDG-04), showed the number of marketable spikes ranging between 1.5 and 2.5. Forty cross lines recorded high number of marketable spikes/ mother corm as opposed against that of check genotype (ARSDG-04) with 1.5 marketable spikes/mother corms.

Days to the seventh floret wilting: It was found variation from 115.00 to 141.00 days with the mean value of 125.15 days. Ten inter-varietal cross lines reflected the seventh floret wilting of more than 130 days after planting whilst eight inter-varietal cross lines and one check genotype (ARSDG-04) reflected the seventh floret wilting in fewer than 120 days after planting. The seventh floret wilting was found between 120 and 130 days in the rest of 28 inter-varietal cross lines or in other words, twenty eight cross lines revealed more earliness than that of check genotype (ARSDG-04) in respect of the seventh floret wilting. The performances of 46 inter-varietal cross lines and one check genotype (ARSDG-04) on flower life (days) in field condition, number of capsules/spike, number of filled capsules/ spike, number of unfilled capsules/spike and total number of capsules /spike is presented in Table 4.

Flower life (days) in the field condition: It varied between 7.00 and 10.60 days having the mean value of 8.56 days. Eleven inter-varietal cross lines revealed 
the maximum range of flower life with more than 9.00 days whereas eleven inter-varietal cross lines revealed the minimum range of flower life with fewer than 8.00 days. On the other hand, twenty four inter-varietal cross lines and one check genotype (ARSDG-04) revealed the flower life between 8.00 and 9.00 days. To sum up, eleven cross lines recorded longer flower life than that of check genotype (ARSDG-04) whereas six inter-varietal cross lines viz., HRSDG-2013-1.2(A), HRSDG-20131.2(B), HRSDG-2013- 2.13(A), HRSDG-2013-7.8 (B), HRSDG-2013-10.18 and HRSDG-2013-11.21 recorded the same flower life period (9 days) as that of check genotype (ARSDG-04) with nine days. On the contrary, check genotype (ARSDG-04) recorded shorter flowerperiod than those of eleven inter-varietal cross lines.

Number of filled capsules/spike: It was variable between 0.00 and 10.00 with the mean value of $4.57 /$ spike. More than 6.00 filled capsules /spike was in 12 inter-varietal cross lines and one check genotype (ARSDG-04) whereas none of the filled capsules was in 3 intervarietal cross lines viz.,HRSDG-2013-10.4,HRSDG2013-11.17(B-1) and HRSDG-2013-11.17(B-2). Fewer than 3.00 filled capsules/spike were in 10 inter-varietal cross lines viz., HRSDG-2013-11.2(B), HRSDG-201311.37(B), HRSDG-2013-11.33, HRSDG-2013-11.21, HRSDG-2013-6.7(A), HRSDG-2013-7.4(B), HRSDG-2013-8.5(B),HRSDG-2013-6.5(A-2), HRSDG-2013-9.7 and HRSDG-2013-10.24. The rest of 21 inter-varietal cross lines recorded between 3.00 and 6.00 filled capsules per spike. The number of filled capsules (9/spike) was similar in check genotype (ARSDG-04) and HRSDG-2013-11.4(B), and only one cross line was able to produce more number of filled capsules (10/spike) than that of check genotype (ARSDG-04) with 9 capsules/spike. In contrast, check genotype (ARSDG-04) produced higher number of filled capsules/spike than those of forty four cross lines.

Table 4 : Performance of 46 inter varietal crossed lines and check genotype of gladiolus for flower life (days), number of filled capsules, number of unfilled capsules and total number of capsules/spike in 2017/2018 at RARS, Lumle.

\begin{tabular}{|c|l|c|c|c|c|}
\hline $\begin{array}{c}\text { Plot } \\
\text { number }\end{array}$ & Inter-varietal cross lines & $\begin{array}{c}\text { Flower life } \\
\text { days) in field } \\
\text { condition }\end{array}$ & $\begin{array}{c}\text { Number of } \\
\text { filled capsules/ } \\
\text { spike }\end{array}$ & $\begin{array}{c}\text { Number of un- } \\
\text { filled capsules/ } \\
\text { spike }\end{array}$ & $\begin{array}{c}\text { Total number } \\
\text { of capsules/ } \\
\text { spike }\end{array}$ \\
\hline 1 & HRSDG-2013-1.2(A) & 9.00 & 4.00 & 1.80 & 5.80 \\
\hline 2 & HRSDG-2013-1.2(B) & 9.00 & 3.80 & 2.40 & 6.20 \\
\hline 3 & HRSDG-2013-1.6(D) & 9.40 & 4.25 & 2.50 & 6.75 \\
\hline 4 & HRSDG-2013-1.14(B) & $\mathbf{1 0 . 3 0}$ & 4.00 & 1.00 & 5.00 \\
\hline 5 & HRSDG-2013-1.14(D) & 8.20 & 5.40 & 2.20 & 7.60 \\
\hline 6 & HRSDG-2013-1.8(D) & 7.75 & 7.50 & 3.00 & 10.50 \\
\hline 7 & HRSDG-2013-2.15(C) & 8.00 & 6.20 & 1.20 & 7.40 \\
\hline 8 & HRSDG-2013-2.13(A) & 9.00 & 6.00 & 0.50 & 6.50 \\
\hline 9 & HRSDG-2013-2.4(D) & 7.20 & 7.20 & 1.80 & 9.00 \\
\hline 10 & HRSDG-2013-2.17(A) & 8.00 & 7.00 & 4.20 & 11.20 \\
\hline 11 & HRSDG-2013-6.17(E) & 8.00 & 7.25 & 3.00 & 10.25 \\
\hline 12 & HRSDG-2013-6.5(A-2) & 8.00 & 1.50 & 0.50 & 2.00 \\
\hline 13 & HRSDG-2013-6.7(A) & 7.60 & 1.00 & 0.00 & 1.00 \\
\hline 14 & HRSDG-2013-6.7(B) & 7.80 & 6.00 & 2.00 & 8.00 \\
\hline 15 & HRSDG-2013-6.5(A-1) & 8.50 & 5.00 & 2.00 & 7.00 \\
\hline 16 & HRSDG-2013-7.4(B) & 10.00 & 1.00 & 0.33 & 1.33 \\
\hline 17 & HRSDG-2013-7.25(A) & 7.25 & 7.60 & 2.40 & 10.00 \\
\hline 18 & HRSDG-2013-7.8(A) & 8.57 & 7.60 & 1.20 & 8.80 \\
\hline 19 & HRSDG-2013-7.8(B) & 9.00 & 7.50 & 1.00 & 8.50 \\
\hline 20 & HRSDG-2013-8.23(A) & 8.20 & 9.50 & 1.75 & 11.25 \\
\hline 21 & HRSDG-2013-8.16(B) & 8.00 & 4.00 & 2.00 & 6.00 \\
\hline 22 & HRSDG-2013-8.5(B) & 8.20 & 1.00 & 0.66 & 1.66 \\
\hline
\end{tabular}




\begin{tabular}{|c|c|c|c|c|c|}
\hline $\begin{array}{c}\text { Plot } \\
\text { number }\end{array}$ & Inter-varietal cross lines & $\begin{array}{c}\text { Flower life } \\
\text { (days) in field } \\
\text { condition } \\
\end{array}$ & $\begin{array}{c}\text { Number of } \\
\text { filled capsules/ } \\
\text { spike }\end{array}$ & $\begin{array}{c}\text { Number of un- } \\
\text { filled capsules/ } \\
\text { spike }\end{array}$ & $\begin{array}{c}\text { Total number } \\
\text { of capsules/ } \\
\text { spike }\end{array}$ \\
\hline 23 & HRSDG-2013-8.5(A) & 8.60 & 3.00 & 2.00 & 5.00 \\
\hline 24 & HRSDG-2013-8.16(A) & 9.60 & 6.00 & 3.00 & 9.00 \\
\hline 25 & HRSDG-2013-8.22(A) & 8.75 & 6.00 & 2.00 & 8.00 \\
\hline 26 & HRSDG-2013-9.12(A-2) & 9.50 & 4.40 & 1.60 & 6.00 \\
\hline 27 & HRSDG-2013-9.12(A) & 7.00 & 6.75 & 1.00 & 7.75 \\
\hline 28 & HRSDG-2013-9.3(D) & 9.60 & 6.00 & 1.33 & 7.33 \\
\hline 29 & HRSDG-2013-9.22(A) & 9.25 & 5.00 & 1.50 & 6.50 \\
\hline 30 & HRSDG-2013-9.7(A) & 8.66 & 2.33 & 2.33 & 4.66 \\
\hline 31 & HRSDG-2013-9.22(E) & 10.00 & 4.00 & 4.33 & 8.33 \\
\hline 32 & HRSDG-2013-9.16(A) & 7.30 & 5.80 & 3.00 & 8.80 \\
\hline 33 & HRSDG-2013-10.24 & 8.50 & 2.66 & 1.00 & 3.66 \\
\hline 34 & HRSDG-2013-10.4 & 9.60 & 0.00 & 0.00 & 0.00 \\
\hline 35 & HRSDG-2013-10.18 & 9.00 & 6.00 & 3.00 & 9.00 \\
\hline 36 & HRSDG-2013-10.5(A) & 9.50 & 3.00 & 0.50 & 3.50 \\
\hline 37 & HRSDG-2013-10.45 & 10.60 & 10.00 & 3.00 & 13.00 \\
\hline 38 & HRSDG-2013-11.2(B) & 8.30 & 1.00 & 1.00 & 2.00 \\
\hline 39 & HRSDG-2013-11.37(B) & 8.00 & 1.00 & 0.00 & 1.00 \\
\hline 40 & HRSDG-2013-11.33 & 7.80 & 1.00 & 1.00 & 2.00 \\
\hline 41 & $\begin{array}{l}\text { HRSDG-2013- } \\
11.17(\mathrm{~B}-1)\end{array}$ & 8.50 & 0.00 & 0.00 & 0.00 \\
\hline 42 & $\begin{array}{l}\text { HRSDG-2013- } \\
11.17(\mathrm{~B}-2)\end{array}$ & 7.50 & 0.00 & 0.00 & 0.00 \\
\hline 43 & HRSDG-2013-11.7(B) & 7.20 & 4.33 & 0.33 & 4.66 \\
\hline 44 & HRSDG-2013-11.21 & 9.00 & 1.66 & 1.33 & 3.00 \\
\hline 45 & HRSDG-2013-11.4(B) & 7.75 & 9.00 & 1.33 & 10.33 \\
\hline 46 & HRSDG-2013-11.42 & 8.50 & 3.00 & 2.5 & 5.50 \\
\hline 47 & ARSDG-04/Check & 9.00 & 9.00 & 1.50 & 9.50 \\
\hline \multicolumn{2}{|r|}{ Minimum } & 7.00 & 0.00 & 0.00 & 0.00 \\
\hline \multicolumn{2}{|r|}{ Mean } & 8.56 & 4.67 & 1.62 & 6.35 \\
\hline
\end{tabular}

\begin{tabular}{|c|c|c|c|c|c|}
\hline $\begin{array}{c}\text { Plot } \\
\text { number }\end{array}$ & Inter-varietal cross lines & $\begin{array}{c}\text { Flower life } \\
\text { (days) in field } \\
\text { condition }\end{array}$ & $\begin{array}{c}\text { Number of } \\
\text { filled capsules/ } \\
\text { spike }\end{array}$ & $\begin{array}{c}\text { Number of un- } \\
\text { filled capsules/ } \\
\text { spike }\end{array}$ & $\begin{array}{c}\text { Total number } \\
\text { of capsules/ } \\
\text { spike }\end{array}$ \\
\hline Maximum & 10.60 & 10.00 & 4.33 & 13.00 \\
\hline CV\% & 10.33 & 60.17 & 67.56 & 63.25 \\
\hline
\end{tabular}

Number of unfilled capsules/spike: It ranged from 0.00 to 4.33 per spike with the mean value of 1.62 per spike. Twelve inter-varietal cross lines produced more than 2.00 unfilled capsules/spike whilst five inter-varietal cross lines produced none of the unfilled capsules/spike. The remaining 29 inter-varietal cross lines produced the number of capsules between 0.5 and 2.00 per spike. Twenty six cross lines produced the fewer number of unfilled capsules/spike that of check genotype (ARSDG-04) whereas twenty cross lines produced higher number of unfilled capsules/ capsule than that of check genotype (ARSDG-04).The higher number of unfilled capsules is most presumably due to the presence of poor stigmatic receptivity in female reproductive organ, non-viable pollen in male reproductive organ and unfavorable weather conditions (excessive rainfall) during pollination.

Total number of capsules/spike:It varied from 0.00 to 13.00 having the mean value of $6.35 /$ spike. A total of 
21 inter-varietal cross lines and one check genotype (ARSDG-04) produced maximum number of capsules (more than 7.00 capsules/spike) whereas three intervarietal cross lines produced none of the capsules/spike. Eight inter-varietal cross lines produced the number of capsules between 1.00 and 3.00 per spike; however, the rest of 13 inter-varietal cross lines produced the number of capsules between 3.00 and 7.00 per spike. check genotype (ARSDG-04) did not produce so high number of capsules as those of six inter-varietal cross lines viz., , HRSDG-2013-1.8(D), HRSDG-2013-2.17(A), HRSDG-2013-6.17(E), HRSDG-2013-8.23(A), HRSDG-2013-10.45 and HRSDG-2013-10.33 while check genotype (ARSDG-04) produced higher number of capsules/spike than those of remaining forty intervarietal cross lines. This result indicates that check genotype (ARSDG-04) consistently possesses genetic inheritance for offering maximum number of capsules with viable seeds which can be utilized as either female parent or male parent in both inter-specific and intervarietal hybridization of gladiolus in future(Poon et al., 2012).

\section{Discussion:}

Values of all sixteen characters contribute to quantitative attributes of all observed 46 inter-varietal cross lines and one check genotype. The inherited traits such as spike emergence, first floret loosening, florets opening at a time, flower diameter and flower life in the field condition determines the peak period of flower harvesting and marketing according to early, main and late season. Plant height, spike length, rachis length, number of florets and floret diameter may assist in choosing the tested lines suited for cut flower, garden display and flower bouquet depending on floret numbers, spike length, flower size and number of flower opening at a time. The number of filled capsules and spikes/mother corm is likely to predict viable lines for maximum propagule (corm and cormel) production (Poon et al, 2017).

The mean value of days taken to the first spike emergence in seven genotypes were nearly 25.00 days earlier in HRS (Horticulture Research Station), Dailekh than those of forty six inter-varietal cross lines and one check/control when planted in the same season of different years in RARS, Lumle(Poon et al., 2017; RARS Annual Report, 2017/018). The differences in the mean value of this character were presumed to have been more effects of climatic conditions than those of genotypes on account of sub-tropical climate in HRS, Dailekh located at an altitude of $1250 \mathrm{~m}$ and mild-temperate climate in RARS, Lumle located at an altitude of $1600-1750$ m in Kaski District.

The mean value of days taken to complete spike emergence in seven genotypes were nearly 23.00 days earlier in HRS (Horticulture Research Station), Dailekh than those of forty six inter-varietal cross lines and one check/control when planted in the same season of different years in RARS, Lumle(Poon et al., 2017; RARS Annual Report, 2017/018 ). The differences in the mean value of this character were presumed to have been more effects of climatic conditions than those of genotypes on account of sub-tropical climate in HRS, Dailekh located at an altitude of $1250-1350 \mathrm{~m}$ and mild-temperate climate in RARS, Lumle located at an altitude of $1600-1750 \mathrm{~m}$ in Kaski District. Cut flowers can be harvested from the mid-spring season till the beginning of monsoon when planted in the first week of February under Dailekh condition. On the other hand, cut flowers can be obtained from late spring season till first fortnight of monsoon season when planted in the same time as in Dailekh under Lumle condition.

The mean value of days taken to the first floret loosening of seven genotypes was found about 22 days earlier in HRS (Horticulture Research Station), Dailekh condition than those of forty six inter-varietal cross lines and one check/control when planted in the same season of different years in RARS, Lumle (Poon et al., 2017; RARS Annual Report, 2017/018 ). The differences in the mean value of this character were presumably due to more effects of climatic and soil conditions than those of genotypes. The annual rainfall varies from $1350 \mathrm{~mm}$ to $1,500 \mathrm{~mm}$ :Temperatures varies from $4{ }^{\circ} \mathrm{C}$ to $34{ }^{\circ} \mathrm{C}$, and soil type is clay loam under Dailekh condition where annual rainfall varies from $5000 \mathrm{~mm}$ to $5500 \mathrm{~mm}$ : Temperature varies from $20 \mathrm{C}$ to $270 \mathrm{C}$, and soil type is sandy loam under Lumle condition (RARS Annual Report, 2017/018 ).

The maximum number of floret opening at a time was 5.0 when thirteen genotypes were evaluated in Nadia District of West Bengal, India (Debnath et al., 2004) while the same character reported by (Poon et al., 2017; RARS Annual Report, 2017/018) was 6.0 in Dailekh, Nepal, but its mean value recorded as 7.0 in the present preliminary study which reveled greater mean value as 
opposed to those of both previous studies in this regard.

The mean spike length of nine gladiolus hybrids showed only $74.00 \mathrm{~cm}$ under coastal Andhra Pradesh condition, India (Naresh et al., 2015). On the other hand, the mean value of the same character of seven genotypes revealed $102.90 \mathrm{~cm}$ under Dailekh condition, Nepal (Poon et al., 2017). Furthermore, the mean value of forty six inclusive of one check genotypes recorded $106.90 \mathrm{~cm}$ spike length in the present preliminary trial (RARS, Annual Report, 2017/018). The findings in these similar experiments reflected smaller differences between the result of HRS, Dailekh and that of RARS, Lumle, Nepal where as bigger differences between the result of coastal AndhraPradesh, India and that of RARS, Lumle, Nepal. Such results were most possibly due to variation in climates,soil fertility, genotypes and time of observations in this regard.

The mean rachis length of nine gladiolus hybrids showed only $38.75 \mathrm{~cm}$ under coastal Andhra Pradesh condition, India (Naresh et al., 2015). On the other hand, the mean value of the same character of seven genotypes revealed $58.30 \mathrm{~cm}$ under Dailekh condition, Nepal (Poon et al., 2017). Furthermore, the mean value of forty six inclusive of one check genotypes recorded $72.50 \mathrm{~cm}$ spike length in the present preliminary trial (RARS, Annual Report, 2017/018). The findings in these similar experiments reflected narrower differences between the result of HRS, Dailekh and that of RARS, Lumle, Nepal whereas wider differences between the result of coastal AndhraPradesh, India and that of RARS, Lumle, Nepal. Such results were most possibly due to differences in climates, soil fertility, genotypes and time of observations in this regard.

The meanvalue of floret diameter $(8.30 \mathrm{~cm})$ in the present trial nearly corroborates the value of the same character $(8.46 \mathrm{~cm})$ recorded by Safeena and Thangam (2019) under Goa condition, India, but somewhat more differences between the result of the same character with $8.04 \mathrm{~cm}$ under Dailekh condition and that $(8.30$ $\mathrm{cm}$ ) of Lumle, condition, Nepal:Such results were most possibly due to differences in soil fertility, genotypes, flower opening stage and time of observations in this regard.

The result of the character (plant height) in this study are moreor lessin conformity with the findings of Poon et al,( 2017) as they reported the mean plant height
$(115.36 \mathrm{~cm})$ of seven genotypes under Dailekhcondition, Nepal. The findings of Chaurasia et al, (2015) reported mean plant height $(107.43 \mathrm{~cm})$ of ten varieties under Gujarat condition of India, which is somewhat smaller value of plant height, and does not fully agree with the finding (119.86 cm) of present study of RARS, Lumle, (2017/018). The variation in plant height of different gladiolus varieties in different sites of Nepal and India might be due to the differential genetic makeup and varied growth rate under various climatic conditions.

The mean number of spikes (2.76/mother corm) is comparatively high in this trial as opposed to those obtained as 2.30, 2.25, 1.69 and 1.43/ mother corm by Safeena and Thangam (2019), Debnath et al, (2004), Poon et al, (2017) and Naresh et al, (2015). In gladiolus, the number of spikes per mother corm depends on number of shoots per plant, which also decides the number of daughter corms per mother corm (Shiramagondi and Hanamashetti, 1999). Spike yield per mother corm is the yield parameter, which is counted as the dependent character on so many independent characters spreading across vegetative and reproductive parameters of these genotypes (Naresh et al., 2015).

Zubair et al, (2013) obtained the average marketable spike (0.9/mother corm) under Pakistan condition; Poon et al, (2017) obtained 1.69 spikes/mother corm under Dailekh condition, Nepal; and the present study showed 2.12 marketable spikes/mother corm under Lumle condition of Nepal, which ismore promising results as compared to those of the findings reported by both authors viz., Zubair et al, (2013) and Poon et al, (2017). This finding is presumably due to transmission of a few heritable traits from parental lines to intervarietal lines so far coupled with suitable environmental conditions of Lumle in this regard.

The observation of flower life in field condition is the milestone criteria to discover promising lines to study vase life in advanced replicated trial. The flower life in field condition and vase life of twenty one genotypes reflected nearly the similar mean results (7.65 and 7.40 days respectively) under Bangalore condition, India (Poon and Rao, 2009). Taye et al, (2015) obtained the result of postharvest-life (9.94 days) whereas Shravan Kumar (2017) evaluated fourteen genotypes of gladiolus for postharvest- life in the ambient condition. The postharvest- life (days) in his study ranged from 
5.94 to 8.26 days with the mean value of 7.10 days under Chhattisgarh condition, India. The present study revealed flower life (8.56 days) which is shorter than that of the former study and longer than that of latter study. Variation in flower life and postharvest-life may be attributed to different environment of field and ambient condition, differential accumulation of carbohydrates from varied leaf production, sensitivity of cultivars to ethylene and genetically framework of the plant. It may also differ due to hereditary character of the cultivars (Shravan Kumar, 2017).

\section{Conclusion:}

The cultivars:American Beauty, Intrepid, Ginger Red, unidentified genotype, Summer Sunshine, White Prosperity and one wild genotype (Pscittacinussp.) were incorporated as parental lines into the intervarietal crossing work in order to develop these forty six inter-varietal lines. These forty six inter-varietal lines have been selectively reduced to twenty five intervarietal lines on the ground of almost all vegetative and floral traits being more desirable than those of parental lines and rest of inter-varietal cross lines. The selected inter-varietal lines are: HRSDG-2013-1.2(A),HRSDG2013-1.14(B), HRSDG-2013-1.14 (D), HRSDG-20132.13(A),HRSDG-2013-1.15(C),HRSDG-2013-2,17 (A),HRSDG-2013-6.17(E),HRSDG-2013-6.7 (A),HRSDG-2013-6.7(B), HR SDG-2013-7.4 (B), HRSDG-2013-7.8(A), HR SDG-2013-7.8 (B),HRSDG-2013-8.5(B),HRSDG-2013-9.12 (A-2),HRSDG-2013-11.7(B),HRSDG-2013-11.17 (B),HRSDG-2013-11.17(B-1),HRSDG-2013-11.17 (B-2),HRSDG-2013-11.33,HRSDG-2013-11.37 (B),HRSDG-2013-9.22(A),HRSDG-2013-8.16(A), HRSDG-2013-9.22(E),HRSDG-2013-10.5(A) and HRSDG-2013-10.2: They need to be further assessed once more year in non-replicated trial so as to advance for replicated trial after the fifth phase of selection. On the ground of overall performances and multiplicative capacity of propagules, only ten intervarietal cross lines: HRSDG-013-2.17(A),HRSDG013-7.4(B), HRSDG-013-7.8(A),HRSDG-013-7.8(B), HRSDG-013-8.5(A),HRSDG-013-8.16(A), HRSDG013-9.12(A-2),HRSDG-013-10.24, HRSDG-01311.7(B)andHRSDG-013-11.17(B-2) along with check genotype are likely to be promoted in replicated trial in order to develop new desirable varieties of gladiolus in future.

\section{Declaration of conflict of interest and ethical approval:}

The authors declare that they don't have any competing interests on the published materials.

\section{References:}

Bhajantri, A., \& Patil, V. S. (2013). Flowering attributes of gladiolus (Gladiolus hybrids hort) genotypes. BIOINFOLET-A Quarterly Journal of Life Sciences, 10(1a), 54-54.

Barman, D., Rajni, K., Pal, R., \&Upadhyaya, R. C. (2005). Corm multiplication of gladiolus as influenced by application of potassium and spike removal. Journal of ornamental Horticulture, 8(2), 104-107.

Kole, C. (Ed.). (2014). Wild crop relatives: genomic and breeding resources: plantation and ornamental crops. Springer Berlin.

Chourasia, A. N. K. I. T., Viradia, R. R., Ansar, H., \& Madle, S. N. (2015). Evaluation of different gladiolus cultivars for growth, flowering, spike yield and corm yield under Saurashtra region of Gujarat. The Bioscan, 10(1), 131-134.

Debnath, M., Basu, D. \& Goswami K. K. (2004). Performance Evaluation of some new Cultivars of Gladiolus on the basis of Farmer's Preference and Market Acceptance in Nadia District, West Bengal. International Conference on Agriculture, Food Science, Natural Resource Management and Environmental Dynamics.

Duncan, M. G. (2000). Grow bulbs: a guide to the species cultivation and propagation of South African Bulbs, National Botanical Institute, Kirstenbosch Gardening Series. Claremont, S. Africa.

Goldblatt, P. (1996). Gladiolus in tropical Africa. Systematics, biology and evolution. Timber Press, Inc.

Goldblatt, P. \& Manning, J. (1998). Gladiolus in southern Africa.Fernwood Press (Pty) Ltd.

Kumar, R., \& Yadav, D. S. (2005).Evaluation of gladiolus cultivars under sub-tropical hills of Meghalaya. Journal of ornamental Horticulture, 8(2), 86-90.

Kamble, B. S., Reddy, B. S., Patil, R. L. T., \& Kulkarni, B. S. (2004).Performance of gladiolus 
(Gladiolus hybridus Hort.) cultivars for flowering and flower quality. Journal of ornamental Horticulture, 7(3and4), 51-56.

Manning, J., Goldblatt, P.,\& Snijman, D. (2002). The color encyclopedia of Cape Bulbs. Timber Press.

Naresh, S., Rao, A. V. D., Bhaskar, V. V., Krishna, K. U., \&Rao, M. P. (2015). Evaluation of gladiolus (Gladiolus hybrida L.) hybrids under coastal Andhra Pradesh conditions. Plant Archives, 15(1), 451-454.

Ohri, D., \&Khoshoo, T. N. (1983). Cytogenetics of garden gladiolus IV.Origin and evolution of ornamental taxa. In Proceedings of the Indian National Science Academy (India).

Poon, T. B. (2009). Seed setting in inter-varietal and inter-specific crosses of Gladiolus (Doctoral dissertation, University of Agricultural Sciences).

Poon, T. B., Pokhrel, A., Shrestha, S., Sharma, S. R., Sharma, K. R. \& Dev, M. B. (2012). Influence of inter-varieNepalese Horticulture, 17(1), 17-24.

RARS Lumle. (2018). Annual Report of Regional Agricultural Research Station, Lumle 2017-18.

Satal and inter-specific crosses on seed set of gladiolus under mid-hill environments of Dailekh condition. Nepal Journal of Science and Technology, 13(1), 17-24.

Poon, T. B., Oli, O.B. \& Chalise, B. (2017).Evaluation of Promising Genotypes of Gladiolus for Important Vegetative and Floral Traits under Mid-hill Environment of Dailekh. Journal of feena, S.A. \&Thangam, M. (2019).Field performance of gladiolus cultivars for growth, yield and quality cut flower production under humid agro climatic conditions of Goa.International Journal of Agriculture Sciences, 11 (3), 7797-7800.

Shaukat, S., Syed, S. H. A. H., \& Shaukat, S.W. (2013). Performance of gladiolus (Gladiolus grandiflora L.) cultivars under the climatic conditions of Bagh Azad Jammu and Kashmir Pakistan. Journal of Central European Agriculture, 14(2), 636-645.

Shiramagond, M. S., \& Hanamashetti, S. I. (1999). Evaluation of varieties in gladiolus under Ghataprabha command area. Karnataka Journal of Agricultural Sciences, 12(1/4), 159-163.

Kumar, S. (2017). Performance of different Gladiolus cultivars on growth, flowering and corm production under Chhattisgarh plains condition (Doctoral dissertation, Indira Gandhi KrishiVishwavidhyalaya, Raipur).

Taye, M.G., Nagera, S.A., Gamada, I. \& Tadese, F.O. (2015). A study of the performance of two prominent cultivars of Gladiolus grandiflorus and to find the effect of three planting dates on growth, production and quality of flowers. African Journal of Wood Science and Forestry, 3(7), 165-169.

Zubair, M., Ayub, G., Amin, N.U., Rab, A., Ahmad, M. $\&$ Ara, N. (2013). Yield quality of cut flowers and corm production of gladiolus vary by cultivar at three levels of nitrogen fertility. Pakistan Journal of Botany, 45(3): 967-976. 